\title{
Correction to: MicroRNA-325-3p protects the heart after myocardial infarction by inhibiting RIPK3 and programmed necrosis in mice
}

Dong-Ying Zhang ${ }^{1 \dagger}$, Bing-Jian Wang ${ }^{1 \dagger}$, Min Ma $^{2}$, Kun Yu ${ }^{1}$, Qing Zhang ${ }^{1}$ and Xi-Wen Zhang ${ }^{1 *}$

\section{Correction to: BMC Molecular Biol (2019) 20:17}

https://doi.org/10.1186/s12867-019-0133-z

The original article [1] contains an error whereby Fig. 7 displays incorrect results; the correct version of Fig. 7 can be viewed ahead in this Correction article and should be considered in place of the original article's version of Fig. 7.

\footnotetext{
*Correspondence: zhangxiwen303@163.com

${ }^{\dagger}$ Dong-Ying Zhang and Bing-Jian Wang are first co-authors

1 Department of Cardiology, The Affiliated Huaian No.1 People's Hospital

of Nanjing Medical University, No.1 West Huanghe Road, Huaiyin District, Huaian 223300, Jiangsu, China

Full list of author information is available at the end of the article
} 
a

TargetScanHuman 7.2 miR-325-3p and position 24-30 of RIPK3 3' UTR

RIPK3-3' UTR-wt

mmu-miR-325-3p

RIPK3-3' UTR-mut
5'-CCCAUCUCCUUAAAGUCAAUAAA-3'

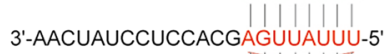

DI+K+1
C

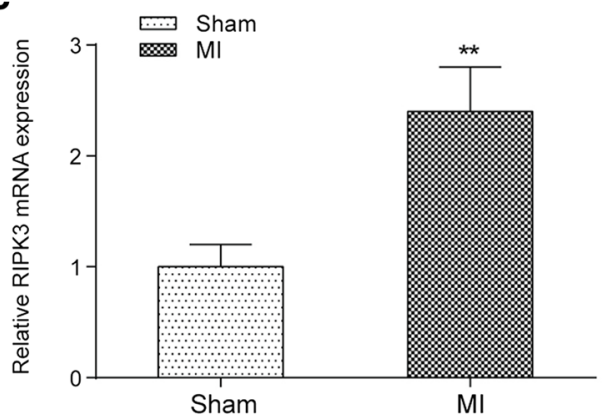

e

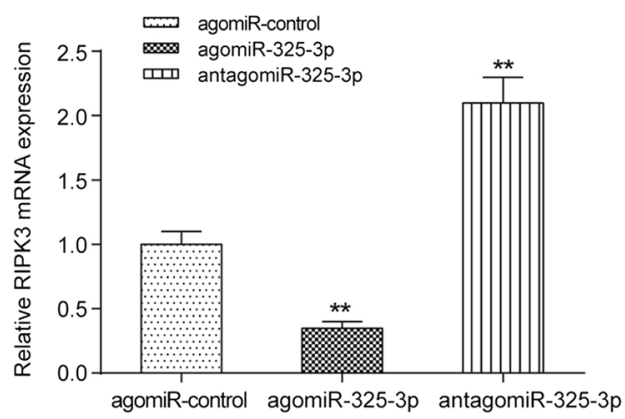

b

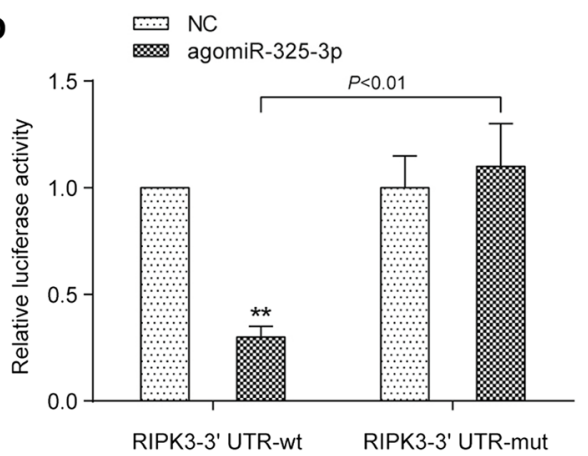

d

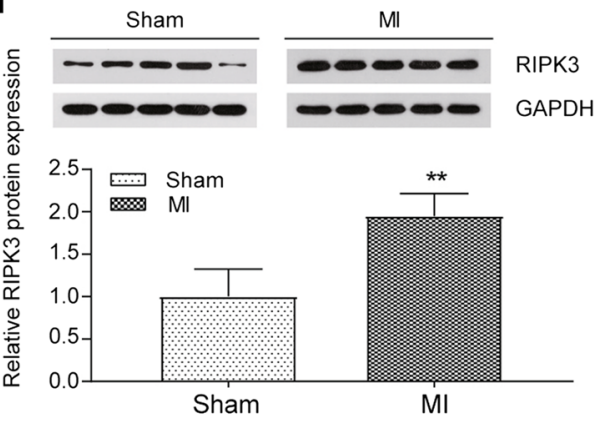

f

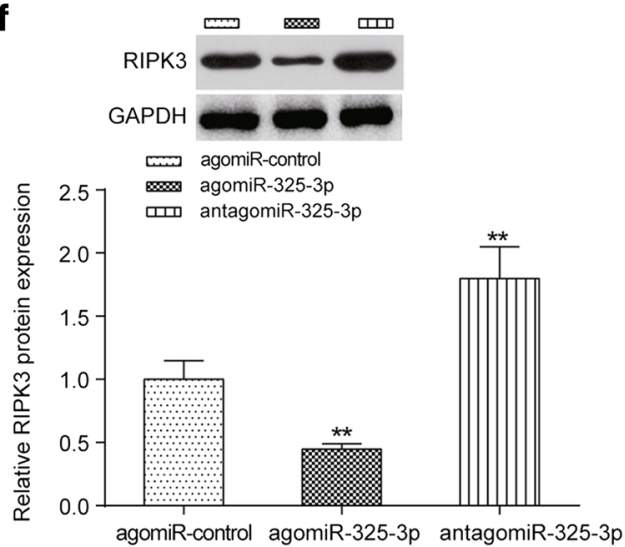

Fig. 7 Target relationship between miR-325-3p and RIPK3. a The binding sites between the $3^{\prime} U T R$ of RIPK3 and miR-325-3p predicted by TargetScanHuman 7.2. b A dual-luciferase reporter assay validated the target relationship between miR-325-3p and the $3^{\prime} U$ TR of RIPK3. ${ }^{*} P<0.01$ between the wild-type and mutated $3^{\prime}$ UTR of RIPK3. c, d The differential expression of RIPK3 mRNA (c) or protein (d) in the sham-operated mice and the Ml mice. ${ }^{* *} P<0.01$ compared to the mice that received the sham operation. $\mathbf{e}$, $\mathbf{f}$ The influence of miR-325-3p dysregulation on the expression of RIPK3 mRNA (e) and protein (f) in MI mice. ${ }^{* *} P<0.01$ compared to Ml mice treated with agomiR-control. Ml, myocardial infarction; agomiR-325-3p, miR-325-3p agomir; antagomiR-325-3p, miR-325-3p antagomir; agomiR-control, scrambled agomir or antagomir control; RIPK3, receptor-interacting serine/threonine protein kinase 3

\section{Author details}

${ }^{1}$ Department of Cardiology, The Affiliated Huaian No.1 People's Hospital of Nanjing Medical University, No.1 West Huanghe Road, Huaiyin District, Huaian 223300, Jiangsu, China. ${ }^{2}$ Department of Cardiology, The Sixth People's Hospital of Chengdu, Chengdu 610051, China.

Published online: 06 August 2019

The original article can be found online at https://doi.org/10.1186/s1286 7-019-0133-z

\section{Reference}

1. Zhang D-Y, Wang B-J, Ma M, Yu K, Zhang Q, Zhang X-W. MicroRNA-325-3p protects the heart after myocardial infarction by inhibiting RIPK3 and programmed necrosis in mice. BMC Mol Biol. 2019;20:17. https://doi. org/10.1186/s12867-019-0133-z.

\section{Publisher's Note}

Springer Nature remains neutral with regard to jurisdictional claims in published maps and institutional affiliations. 